\title{
A Robust Open-source Algorithm to Detect Onset and Duration of QRS Complexes
}

\author{
W Zong ${ }^{1,2}$, GB Moody ${ }^{1}$, D Jiang ${ }^{3}$ \\ 'Harvard-MIT Division of Health Sciences and Technology, Cambridge, MA, USA \\ ${ }^{2}$ Beth Israel Deaconess Medical Center, Harvard Medical School, Boston, MA, USA \\ ${ }^{3}$ Institute of Biomedical Engineering, Xi' an Jiatong University, Xi' an, Shaanxi, China
}

\begin{abstract}
This paper presents a novel algorithm to detect onset and duration of $Q R S$ complexes. After low-pass filtering, the ECG signal is converted to a curve length signal by $a$ transform in which a nonlinear scaling factor is introduced to enhance the QRS complex and to suppress unwanted noise. Adaptive thresholds are applied to the length signal to determine the onset and duration of the QRS complex. The algorithm was evaluated with the complete set of single channel ECGs (signal 0) from the MIT-BIH Arrhythmia Database, and achieved a gross QRS sensitivity of $99.65 \%$ and a gross $Q R S$ positive predictive accuracy of $99.77 \%$. The $Q R S$ onset determination is very stable and is insensitive to $Q R S$ morphology change. The noise tolerance of the algorithm was evaluated using the MIT-BIH Noise Stress Test Database. The $C$ source code for the single-channel algorithm has been contributed to PhysioToolkit and is freely available from PhysioNet (www.physionet.org).
\end{abstract}

\section{Introduction}

QRS detection is the first and most crucial step in automatic electrocardiogram (ECG) analyses such as arrhythmia detection and classification, ECG diagnosis, Holter, and heart rate variability (HRV) studies.

Existing QRS detectors typically define the QRS fiducial point as the maximal slope or the peak of the $R$ wave $[1,2]$. The fiducial point will therefore vary with changes in QRS morphology associated with axis shifts and alterations in ventricular depolarization patterns. Such subtle variation may affect the accuracy in HRV analysis or ECG-blood pressure (BP) delay time studies [3]. Most existing QRS detectors do not provide a precise location for the beginning of the ventricular excitation, which is the time of interest in such studies.

Only a few QRS detectors in the literature address the measurement of $Q R S$ duration $[4,5]$, which is a sensitive feature for beat classification and a useful reference for ST-segment measurement.

This article presents a robust algorithm to detect onset and duration of QRS complexes. The algorithm employs and extends the basics of the simplified curve length transform [6]. A nonlinear scaling factor for ECG curve length is introduced to enhance the QRS complex and to suppress other parts of ECG and unwanted noise, which makes the detection of QRS onset and duration from the ECG curve length features possible. The algorithm was initially developed in [7] and was recently reimplemented. Its performance has been evaluated using standard databases $[8,9]$ and methodology [10]. The $\mathrm{C}$ source code of the algorithm has been made freely available via PhysioNet [11].

\section{Methods}

The algorithm consists of three components: a low-pass filter, nonlinearly scaled curve length transformation, and decision rules, as shown in figure 1 .

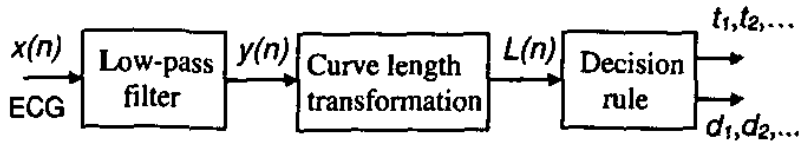

Figure 1. The algorithm overview diagran

The ECG signal, $x(n)$, is the input of the low-pass filter which produces the filtered signal, $y(n)$. The curve length transformation converts $y(n)$ to a curve length signal, $L(n)$. The decision rule is applied to $L(n)$ to determine the time location of QRS onsets, $t_{l}, t_{2}, \ldots$, and corresponding QRS durations, $d_{k}, d_{2}, \ldots$

\subsection{Low-pass filter}

For adult human ECG, the ideal passband for a QRS detector is approximately $5-15 \mathrm{~Hz}[12,13]$. In the present algorithm, only a low-pass filter is necessary, because the 
nonlinearly scaled curve length transformation will substantially suppress low frequency components. Our detector uses a second order recursive low-pass filter as described by Lynn [14]. Formulas (1) and (2) are its transfer and frequency-response functions, respectively,

$$
\begin{aligned}
& H(z)=\frac{\left(1-z^{-5}\right)^{2}}{\left(1-z^{-1}\right)^{2}} \\
& |H(\omega T)|=\frac{\sin ^{2}(3 \omega T)}{\sin ^{2}(\omega T / 2)}
\end{aligned}
$$

where $T$ is the sampling period. The difference equation is:

$$
\begin{array}{r}
y(n)=2 y(n-1)-y(n-2)+x(n) \\
-2 x(n-5)+x(n-10)
\end{array}
$$

where the $3 \mathrm{~dB}$ cut-off frequency is about $16 \mathrm{~Hz}$, for an ECG signal sampled at $250 \mathrm{~Hz}$, and the gain is 25 at $0 \mathrm{~Hz}$. The phase shift is $20 \mathrm{~ms}$ ( 5 samples at $250 \mathrm{~Hz}$ ).

\subsection{Curve length transformation}

According to ECG theory, the electrical activity of the myocardium can be considered to be equivalent to a synthetic electric dipole vector moving in the conductive space. The locus of the end point of the dipole vector constitutes a spatial curve and its projections on each lead axis form the corresponding ECG potentials. When the time, $t$, is considered as the parameter variable and each lead as a spatial coordinate, the spatial curve, $\vec{s}=\vec{s}(t)$, of the movement of the end point of the dipole vector can be completely or partially represented by the ECG potentials in each lead as follows:

$$
\vec{s}=\vec{s}(t)=\left\{y_{1}(t), y_{2}(t), \ldots, y_{n}(t)\right\}
$$

where $y_{i}(t), i=1,2, \ldots, n$, are the ECG potentials on each lead.

It is known that, in certain time windows, the ECG curve length corresponding to the QRS complex is generally longer than that of the other parts with the same time window. If the time window is chosen to be approximately equal to the QRS duration, it can be expected to yield a locally maximal curve length at the QRS location. Thus, the ECG curve length feature can be used for QRS detection.

\section{Length transformation for one-channel ECG}

If $y(t)$ is continuously differentiable over the time interval $[a, b]$, then the length of $y(t)$ in this time interval equals a bounded value $L$ :

$$
L=\int_{a}^{b} d s=\int_{a}^{b} \sqrt{1+\left(\frac{d y}{d t}\right)^{2}} d t
$$

If $w$ is the duration of the time window, the curve length transformation of function $y=y(t)$ over the interval $[t-w, t]$ is defined as:

$$
L(w, t)=\int_{t-w}^{t} \sqrt{1+\left(\frac{d y}{d t}\right)^{2}} d t
$$

where $w<b-a$ and $a+w<t<b$. The discrete form of (6) is as follows:

$$
L(w, i)=\sum_{k=i-w}^{i} \Delta S_{k}=\sum_{k=i-w}^{i} \sqrt{1+\left(\frac{\Delta y_{k}}{\Delta t}\right)^{2}} \Delta t
$$

where $\Delta y_{k}=y_{k}-y_{k-1}$ and $l+w<i \leq N, N$ is the total number of the sample points and $w<1$. Since $\Delta t$ is the sampling period (a constant), the above can be written as:

$$
L(w, i)=\sum_{k=i-w}^{i} \sqrt{\Delta t^{2}+\Delta y_{k}^{2}}=\sum_{k=i-w}^{i} \sqrt{C+\Delta y_{k}^{2}}
$$

where $C=\Delta t^{2} . C$ is a constant, but it can be considered as a nonlinear scaling factor. The effect of the $C$ will be further discussed below.

\section{Length transformation for multi-channel ECG}

The curve length transformation for $\mathrm{n}$-dimensional function, $y_{j}=y_{j}(t), j=1,2, \ldots, n$, is defined as:

$$
L_{n}(w, t)=\int_{t-w}^{t} \sqrt{\sum_{j=1}^{n}\left(d s_{j}\right)^{2}} d t=\int_{t-w}^{t} \sqrt{\sum_{j=1}^{n}\left(1+\left(\frac{d y_{j}}{d t}\right)^{2}\right.} d t
$$

Similar to (8), according to formula (9), we can define the n-channel ECG curve length transformation in discrete form as:

$$
\begin{aligned}
L_{n}(w, i) & =\sum_{k=i-w}^{i} \sqrt{\sum_{j=1}^{n}\left(\Delta t^{2}+\Delta y_{j, k}^{2}\right)} \\
& =\sum_{k=i-w}^{i} \sqrt{\sum_{j=1}^{n}\left(C+\Delta y_{j, k}^{2}\right)}
\end{aligned}
$$

\section{Selection of the window width (w)}

Figure 2 shows the relationship between an idealized QRS complex and its length transform (LT) signal. The ascendant section of the LT signal indicates the duration of QRS complex. In order to extract QRS duration information as well as the QRS location, the window $w$ 
should be approximately equal to the width of the widest QRS complex. For our application, the $w$ is chosen to be $130 \mathrm{~ms}$.

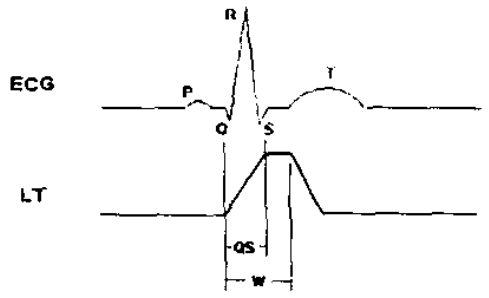

Fig 2. Relationship between QRS complex and the LT signal, where $\mathrm{QS}$ is the QRS duration and $\mathrm{w}$ is the analyzing widow.

\section{The effect of the scaling factor $C$}

The constant $C$ in (8) and (10) is a length-scaling factor. Let us consider the length element in formula (8):

$$
\Delta L_{i}=\sqrt{C+\Delta y_{i}^{2}}
$$

If $l=\Delta L_{i}$ and $u=\Delta y_{i}$, then formula (11) becomes:

$$
l=\sqrt{C+u^{2}}
$$

The effect of a signal difference $u$ on a length element $l$ is given by:

$$
\frac{d l}{d u}=\frac{u}{\sqrt{C+u^{2}}}
$$

We define $d V d u$ as the length response ratio.

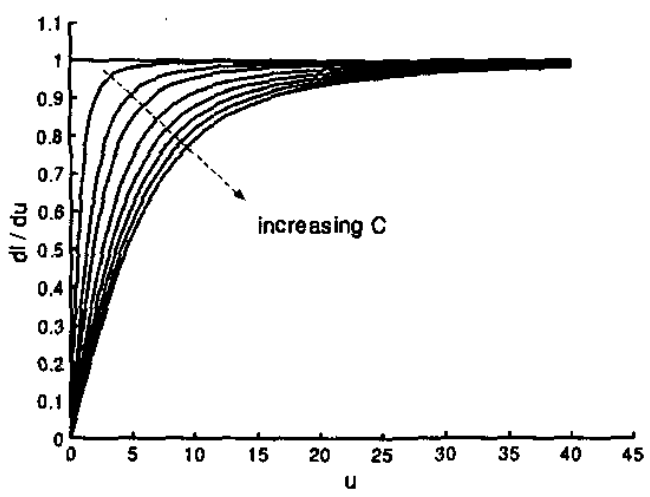

Fig. 3. The effect of the signal difference $u$ on the length transform, for $C=0,1,5,10,20,30,40,50$, and 60 .

Fig. 3 shows the length response ratio for various value of $C$. When $C=0$, the length response ratio is 1.0 for all values of $u$. As $C$ increases, the length response ratio to lower values of $u$ is reduced dramatically and the ratio stays close to 1 at high values of $u$. By selecting a proper $C$ value, the LT signal response to QRS portion can be further enhanced and other parts of ECG such as $P$ wave, $T$ wave, and unwanted noise, especially those near the onset and end of the QRS, can be further suppressed. The $C$ value in the algorithm is determined based on the statistical observations of the signal differential values of QRS complexes, $\mathrm{P}$, and $\mathrm{T}$ waves [7]. The absolute $C$ value is associated with the sampling frequency and sampling resolution of the signal.

Figure 4 shows an example for a single channel ECG. Note the effects of the nonlinearly scaled LT for enhancing QRS and suppressing other parts and noises.

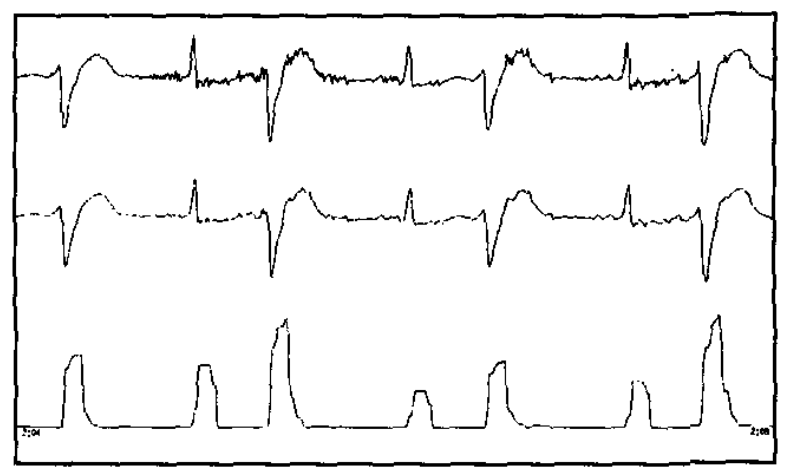

Fig. 4. Example of single channel ECG processing. From top to bottom: the original ECG, the low-pass filtered ECG, and the non-linearly scaled LT signal; 5 seconds per trace.

\subsection{Decision rule}

The decision rule consists of two procedures: (1) thresholding on the LT signal to find a possible QRS position; (2) searching locally to find the QRS onset and duration.

(1) Adaptive thresholding

A threshold base value is established and is initially assigned as three times the mean value of the LT signal for the initial 10 -second period. The actual threshold is set to $1 / 3$ of the threshold base value. The threshold base value is then adaptively adjusted, based on the maximum LT value of each detected QRS complex.

(2) Local search strategies

When the LT signal crosses the threshold, a probable QRS is noted. From the threshold-crossing point, $t c_{i}$, the algorithm searches backward for $125 \mathrm{~ms}$ to get a minimum value, $L \min _{i}$, and forward for $125 \mathrm{~ms}$ to get a maximum value, $L \max _{i}$. The difference, $L A_{i}=L \max _{k}-L \min _{i}$ is obtained. Then, from $t c_{i}$ again, the algorithm searches backward to find the location, $Q b_{i}$, where the $\mathrm{LT}$ value drops monotonically to $L \min _{i}+L A_{j} / 100$, and searches forward to find the location, $S b_{i}$, where the $L T$ increases to $L$ max $i-L A / 20 . \quad Q b_{i}$ and $S b_{i}$ are considered the base values of QRS onset and end, respectively. The actual QRS onset is adjusted by $-20 \mathrm{~ms}$ or -5 samples and the actual end is adjusted by $+20 \mathrm{~ms}$ or +5 samples. This widening adjustment compensates for the time interval 
loss caused by the onset/end thresholds. The adjustments are based on statistical observation of the differences between the algorithm's onset/end estimates and human expert judgments.

Finally, a $250 \mathrm{~ms}$ eye-closing period is applied after each detected QRS to avoid possible double detection of the same beat.

\section{Results}

Evaluated using bxb [10], a standard beat annotation comparison utility freely available from PhysioNet [11], with the complete set of single channel ECGs (signal 0, resampled at $250 \mathrm{~Hz}$ ) from the MIT-BIH Arrhythmia Database [8], this algorithm achieved a gross QRS sensitivity of $99.65 \%$ and a gross QRS positive predictive accuracy of $99.77 \%$.

Representative QRS complexes of 156 normal beats, 82 premature ventricular contractions ( $\mathrm{PVCs}$ ), 23 bundle branch block (BBB) beats, and 22 fusion beats selected from 45 records in the MIT-BIH Arrhythmia Database were used to evaluate the accuracy of the algorithm's determinations of QRS onset and duration. By manual comparison of the QRS onset and end markers produced by the algorithm and those recorded by human experts, the mean difference for the QRS onset was $0.4(+1-2.4)$ $\mathrm{ms}$ for normal beats, $1.4 \mathrm{~ms}(+/-4.9 \mathrm{~ms})$ for PVCs, $6.1 \mathrm{~ms}$ $(+/-8.3 \mathrm{~ms})$ for BBB beats, and $1.8 \mathrm{~ms}(+/-2.4 \mathrm{~ms})$ for fusion beats; the mean difference for the QRS end was $1.4 \mathrm{~ms}(+/-2.4 \mathrm{~ms})$ for normal beats, $1.1 \mathrm{~ms}(+/-2.4 \mathrm{~ms})$ for PVCs, $5.2 \mathrm{~ms}(+/-6.3 \mathrm{~ms})$ for BBB beats, and $0.9 \mathrm{~ms}(+/-$ $2.1 \mathrm{~ms})$ for fusion beats.

The noise tolerance of the algorithm was evaluated using the MIT-BIH Noise Stress Test Database $[9,11]$. Noise had no measurable effects above a signal-to-noise ratio (SNR) of $12 \mathrm{~dB}$, at which QRS positive predictivity dropped from nearly $100 \%$ to $93 \%$; while QRS sensitivity remained at nearly $100 \%$. At an SNR of $6 \mathrm{~dB}$, QRS sensitivity dropped to $99.6 \%$ and QRS positive predictivity dropped to $75 \%$.

\section{Conclusion and discussion}

A novel, effective, and noise-tolerant QRS detection algorithm based on nonlinearly scaled ECG curve length feature has been developed. The QRS onset determination is very stable and is insensitive to QRS morphology change. The accuracy of the QRS duration detection is reasonably satisfactory and would be sufficient for arrhythmia analysis purposes. The algorithm has good tolerance to noise and is successful in rejecting baseline wander and suppressing tall $\mathrm{P}$ or $\mathrm{T}$ waves. The algorithm is easily extendable for analysis of multi-channel ECGs. The $\mathrm{C}$ source code for the singlechannel algorithm has been contributed to PhysioToolkit and is freely available from PhysioNet website (hup://www.physionet.org).

\section{References}

[1] Kohler BU, Hennig C, Orglmeister R. The principles of software QRS detection. IEEE Eng Med Biol Mag 2002; (Jan/Feb):42-57.

[2] Pahlm O, Sornmo L. Software QRS detection in ambulatory monitoring - a review. Med Biol Eng Comput 1984; 22:289. 297.

[3] Zong W, Moody GB, Mark RG. Effects of vasoactive drugs on the relationship between ECG-pulse wave delay time and arterial blood pressure in ICU patients. Computers in Cardiology 1998; 25:673-676.

[4] Engelse WAH, Zeelenberg C. A single scan algorithm for QRS-detection and feature extraction. Computers in Cardiology 1979; 6:37-42.

[5] Jane R, Blasi A, Garcia J, et al. Evaluation of an automatic threshold based detector of waveform limits in Holter ECG with the QT database. Computers in Cardiology 1997; 24:295-298.

[6] Gritzali F. Towards a generalized scheme for QRS detection in ECG waveforms. Signal Processing 1988; 15:183-192.

[7] Zong W. The studies on ECG feature extraction and arrhythmia detection and classification by fuzzy reasoning. Ph.D. dissertation, Xi'an Jiaotong University, Xi'an, China 1993.

[8] Moody GB, Mark RG. The MIT-BIH arrhythmia database on CD-ROM and software for use with it. Computers in Cardiology 1990; 17:185-188.

[9] Moody GB, Muldrow WE, Mark RG. A noise stress test for arrhythmia detectors. Computers in Cardiology 1984; $11: 381-384$.

[10] Moody GB, Feldman CL, Bailey JJ. Standards and applicable databases for long-term ECG monitoring. $J$. Electrocardiology 1993; 26(Suppl):151-155.

[11) Goldberger AL, Amaral LAN, Glass L, et al. PhysioBank, PhysioToolkit, and PhysioNet: components of a new research resource for complex physiologic signals. Circulation 2000; 101(23):e215-e220.

[12] Goovaerts HG, Ros HH, van den Akker TJ, et al. A digital QRS detector based on the principle of contour limiting. IEEE Trans Biomed Eng 1976; BEM-23:154.

[13] Thakor NV, Webster JG, Tompkins WJ. Optimal QRS detector. Med Biol Eng Comput 1983; 21:343-350.

[14] Lynn PA. Online digital filter for biological signals: some fast designs for a small computer. Med Biol Eng Comput $1977 ; 15: 534-540$.

Address for correspondence:

Wei Zong, Ph.D.

Massachusetts Institute of Technology

Room E25-505

77 Massachusetts Avenue

Cambridge, MA 02139, USA

E-mail: wzong@mit.edu 